BUDKERINP/2002-60

\title{
ON THE CALCULATION OF THE NLO VIRTUAL PHOTON IMPACT FACTOR *
}

\author{
V.S. Fadin ${ }^{a, b \dagger}$, D.Yu. Ivanov ${ }^{c, e} \ddagger$ and M.I. Kotsky ${ }^{a} \dagger$ \\ ${ }^{a}$ Budker Institute for Nuclear Physics, 630090 Novosibirsk, Russia \\ ${ }^{b}$ Novosibirsk State University, 630090 Novosibirsk, Russia \\ ${ }^{c}$ Institute of Mathematics, 630090 Novosibirsk, Russia \\ ${ }^{e}$ Regensburg University, Germany
}

\begin{abstract}
The definition of the virtual photon impact factor involves the integration of the $s$-channel discontinuity of the photon-Reggeon scattering amplitude over the right cut. It permits to formulate a new approach for the calculation of the impact factor based on analytical properties of the amplitude in question. In the next-to-leading order it may give a possibility for considerable simplification of the calculation. We have shown that a part of the diagrams contributing to the impact factor can be treated without their real calculation.
\end{abstract}

*Work supported in part by INTAS and in part by the Russian Fund of Basic Researches.

$\dagger^{\dagger}$-mail address: FADIN@INP.NSK.SU

$\ddagger_{e-m a i l ~ a d d r e s s:}$ D-IVANOV@MATH.NSC.RU

${ }^{\dagger \dagger}$ e-mail address: KOTSKY@INP.NSK.SU 


\section{Introduction}

There are at least two reasons why the total cross section of interaction of photons with large virtualities $\sim Q^{2} \gg \Lambda_{Q C D}^{2}$ at high c.m.s. energies $\sqrt{s}$ is an attractive object for theoretical investigation. The first one is that it was experimentally measured (see [1] - [3] and references therein); the second at large enough $Q^{2}$ this cross section can be calculated in a framework of perturbative QCD. The most interesting is the region of so small values of $x=Q^{2} / s$ where, firstly, the main contribution to the cross section is given by nondecreasing with energy terms, and secondly, a resummation of the higher order contributions enhanced by powers of $\log (1 / x)$ is necessary.

The most common basis for such resummation is given by the BFKL approach [4]. This approach in the leading logarithmic approximation (LLA), when only leading terms $\left(\alpha_{s} \ln s\right)^{n}$ are resumed, was extensively used for theoretical analysis of $\gamma^{*} \gamma^{*}$ interaction [5]. Unfortunately, in the LLA neither scale of energy, nor argument of the running coupling constant $\alpha_{s}$ are fixed, so that for accurate theoretical prediction we have to know the radiative corrections to the LLA. Recently, the radiative corrections to the kernel of the BFKL equation had been calculated [6] - [11] and the kernel for the forward scattering is presently known in the next-to-leading order (NLO) $[12,13]$. Attempts to apply it for the description of experiment [14] are encouraging, but for a consistent comparison with the data one needs to know the impact factors of colliding particles with the same accuracy as the kernel of the BFKL equation.

The cross section of interaction of particles $A$ and $B$ is given in the BFKL approach by the convolution of the Green's function $G$ with the impact factors $\Phi_{A}$ and $\Phi_{B}$. The Green's function describes the propagation of two interacting Reggeized gluons, it is determined by the kernel of the BFKL equation. Whereas the impact factors describe the interaction of the colliding particles with the Reggeized gluons.

$$
\sigma_{A B}(s)=\int_{\delta-i \infty}^{\delta+i \infty} \frac{d \omega}{2 \pi i} \int \frac{d^{2} q_{A}}{2 \pi \vec{q}_{A}^{2}} \int \frac{d^{2} q_{B}}{2 \pi \vec{q}_{B}^{2}}\left(\frac{s}{s_{0}}\right)^{\omega} \Phi_{A}\left(\vec{q}_{A}, s_{0}\right) G_{\omega}\left(\vec{q}_{A},-\vec{q}_{B}\right) \Phi_{B}\left(\vec{q}_{B}, s_{0}\right) .
$$

Here the vector sign is used for vector components transverse to the plane of the initial momenta $p_{A}, p_{B}, G_{\omega}$ is the Mellin transform of the Green's function, and $s_{0}$ is an appropriately chosen energy scale. The representation (1.1) is valid both in the LLA and in the NLA. In both cases $G_{\omega}\left(\vec{q}_{A},-\vec{q}_{B}\right)$ is scale-independent. The impact factors become dependent in the NLA on the energy scale in such a way that under variations of $s_{0}$ the cross section remains, within the NLA accuracy, unchanged. It was shown in [15] that to the NLA accuracy one can change the scale $s_{0}$ in (1.1) for any factorizable scale $f_{A} f_{B}$, with $f_{i}$ depending on $\vec{q}_{i}$, without changing the Green's function, provided that the impact factors are also changed correspondingly.

At present impact factors are known in the NLO for the scattering of elementary coloured particles, quarks and gluons $[16,17]$. In the case of the colorless particles scattering the corresponding results for the impact factors have been obtained in the leading order (LO) only. This restricts much a predictive power of the BFKL approach. In [14] 
the phenomenological analysis is performed with use of the $\mathrm{LO} \gamma^{*}$ impact factors ${ }^{1}$, therefore, the theoretical predictions have some spread related to the choice of the energy scale $s_{0}$. To obtain more certain predictions one needs to know the NLO $\gamma^{*}$ impact factors, that makes their evaluation a very important and timely problem.

The knowledge of the NLO $\Phi_{\gamma^{*}}$ is necessary not only at energies at which the BFKL dynamics is completely developed, but also in the case when only a few terms of BFKL series do contribute (that is probably the case at modern energies). In this situation the NLO $\Phi_{\gamma^{*}}$ determines a size of radiative corrections to the non-decreasing with $s$ contribution to the total cross section.

The calculation of $\Phi_{\gamma^{*}}$ in the NLO have been started in [19] - [23]. The impact factors are unambiguously defined [24] in terms of the effective vertices for the Reggeon-particle interactions. For the evaluation of $\Phi_{\gamma^{*}}$ one needs to know the amplitude $\Gamma_{\gamma^{*} R \rightarrow q \bar{q}}^{c}$ for the $q \bar{q}$ - pair production in $\gamma^{*}$-Reggeon collision up to the one-loop accuracy as well as the amplitude at Born level describing the radiation of an additional gluon, $\Gamma_{\gamma^{*} R \rightarrow q \bar{q} g}^{c}$. They presently are both known [20] - [22] and can be used for the calculation of $\Phi_{\gamma^{*}}$ in the NLO. For this purpose the amplitudes must be squared and integrated over the phase space and the squared invariant mass of the produced particles. Unfortunately the corresponding expressions are extremely complicated which makes it difficult to progress further with analytical calculations.

In this situation it is natural to look for an alternative possibility to organize evaluation of $\Phi_{\gamma^{*}}$. In this paper we suggest an approach based on intensive use of analytical properties of Feynman diagrams of an effective field theory with the Reggeized gluon. This method allows to consider part of the diagrams without their real calculation which simplifies considerably the problem.

The paper is organized as follows. In the next section we give a diagrammatic representation for the virtual photon impact factor. In the Sections 3 and 4 the analytical properties of the Feynman diagrams are discussed in length and the simplifications due to the analyticity are derived. The last section contains our conclusions.

\section{Representation of the impact factor}

We use the Sudakov decomposition of the virtual photon and the Reggeon momenta $p_{A}$ and $q$

$p_{A}=p_{1}-\frac{Q^{2}}{s} p_{2}, \quad p_{A}^{2}=-Q^{2}, \quad q=\alpha_{R} p_{2}+q_{\perp}=\frac{\tilde{s}+Q^{2}+\vec{q}^{2}}{s} p_{2}+q_{\perp}, \quad q^{2}=q_{\perp}^{2}=-\vec{q}^{2}$,

where $\left(p_{1}, p_{2}\right)$ is the light-cone basis of the initial particles momenta plane

$$
p_{1}^{2}=p_{2}^{2}=0, \quad 2 p_{1} p_{2}=s \rightarrow \infty,
$$

\footnotetext{
${ }^{1}$ These impact factors differ by only trivial coefficients from the analogous ones in QED, which were obtained many years ago [18].
} 
and $\tilde{s}=\left(p_{A}+q\right)^{2}$ is the virtual photon - Reggeon invariant mass squared. It is of order of typical transverse momenta, i.e., limited and does not grow with $s$ since the impact factor is related by the definition to production of $q \bar{q}$ and $q \bar{q} g$ states in the $\gamma^{*}$ fragmentation region. $^{2}$

The impact factor $\Phi_{\gamma^{*}}$ in the LO, where a Reggeized gluon acts as the ordinary gluon with polarization vector $p_{2} / s$, reads

$$
\Phi_{\gamma^{*}}^{(0)}(\vec{q})=\frac{1}{\sqrt{N_{c}^{2}-1}} \sum_{\{a\}} \int \frac{d \tilde{s}}{2 \pi}\left|\Gamma_{\gamma^{*} R \rightarrow q \bar{q}}^{(0) c}\right|^{2} d \rho_{q \bar{q}} .
$$

Here $N_{c}=3$ is the number of QCD colours, $\Gamma_{\gamma^{*} R \rightarrow q \bar{q}}^{(0) c}$ is the amplitude of $q \bar{q}$ pair production in the $\gamma^{*} R$ collision, evaluated in the Born approximation, $\tilde{s}$ is the squared c.m.s. energy of the $\gamma^{*} R$ system (equal in this case to the invariant mass of the produced $q \bar{q}$ pair), $d \rho_{q \bar{q}}$ is the phase space element of the pair, see Ref. [25]. The sum $\{a\}$ is over all discrete quantum numbers of the produced pair.

The representation of $\Phi_{\gamma^{*}}$ in the NLO is more complicated. First of all, $\Phi_{\gamma^{*}}$ is expressed in terms of $\Gamma_{\gamma^{*} R \rightarrow f}^{c}$ in a more complicated way [24] and depends on the energy scale $s_{0}$ in the Mellin transform (1.1):

$$
\begin{gathered}
\Phi_{\gamma^{*}}\left(\vec{q}, s_{0}\right)=\frac{1}{\sqrt{N_{c}^{2}-1}} \sum_{\{f\}} \int \frac{d \tilde{s}}{2 \pi}\left|\Gamma_{\gamma^{*} R \rightarrow f}^{c}\right|^{2} d \rho_{f} \theta\left(M^{2}-\tilde{s}\right) \\
-\frac{g^{2} N_{c} \vec{q}^{2}}{(2 \pi)^{D-1}} \int \frac{d^{D-2} q_{1}}{\vec{q}_{1}^{2}\left(\vec{q}_{1}-\vec{q}\right)^{2}} \Phi_{\gamma^{*}}^{(0)}\left(\vec{q}_{1}\right) \ln \frac{M^{4}}{s_{0}\left(\vec{q}_{1}-\vec{q}\right)^{2}}-\omega^{(1)}\left(q^{2}\right) \Phi_{\gamma^{*}}^{(0)}(\vec{q}) \ln \frac{\vec{q}^{2}}{s_{0}},
\end{gathered}
$$

where the sum $\{f\}$ is over all discrete quantum numbers of the contributing intermediate states, which are now $q \bar{q}$ and $q \bar{q} g ; M \rightarrow \infty$ is the cut off, which becomes necessary since the integral over $\tilde{s}$ is divergent for the $q \bar{q} g$ state. The dependence on $M$ in the r.h.s of Eq. (2.4) vanishes due to cancellation between the first and the second terms. $D=4+2 \epsilon$ is the space-time dimension taken different from $D=4$ for regularization of infrared and ultraviolet divergences which appear at the intermediate steps. Note that Eq. (2.4) provides the infrared finiteness of the impact factor as it was shown explicitly in Ref. [25]. The last two terms in Eq. (2.4) are to subtract the contribution coming from the emission of a gluon (both the real and the virtual one) outside the fragmentation region of the photon $\gamma^{*}$, the effects which were taken into account already in the LLA.

$$
\omega^{(1)}(t)=-\frac{g^{2} N_{c} \Gamma(1-\epsilon) \Gamma^{2}(\epsilon)}{(4 \pi)^{2+\epsilon} \Gamma(2 \epsilon)}\left(\vec{q}^{2}\right)^{\epsilon}
$$

is the gluon Regge trajectory in the one-loop approximation. In the NLO the Reggeon vertices depend on a scale of energy used in the Reggeon factor (see Ref. [26] for more details). In the vertices $\Gamma_{\gamma^{*} R \rightarrow q \bar{q}}^{c}$ entering in Eq. (2.4) this scale is taken equal $\vec{q}^{2}$.

\footnotetext{
${ }^{2}$ The component of the Reggeon momentum proportional to Sudakov vector $p_{1}$ is irrelevant for the analysis of the impact factor, therefore it is not included in the Eq. (2.1).
} 


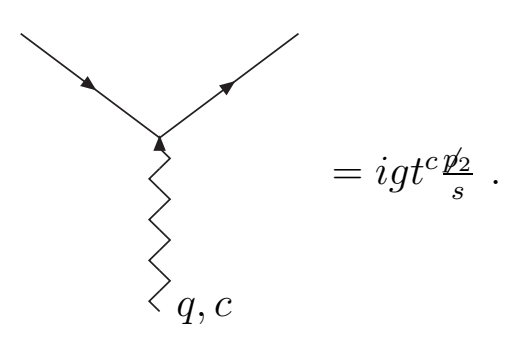

(a)

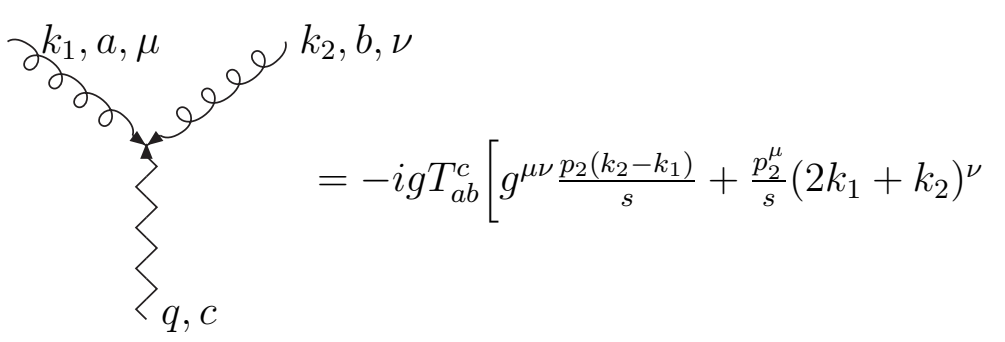

(b)

$$
\left.-\frac{p_{2}^{\nu}}{s}\left(2 k_{2}+k_{1}\right)^{\mu}+\frac{2 q^{2}}{s} \frac{p_{2}^{\mu} p_{2}^{\nu}}{p_{2}\left(k_{1}-k_{2}\right)}\right] \theta\left(\left|\frac{p_{2}\left(k_{1}-k_{2}\right)}{s}\right|-\beta_{0}\right) .
$$

Figure 1: The quark-quark-Reggeon and the gluon-gluon-Reggeon effective vertices. The zig-zag lines represent the Reggeized gluon; $t^{c}$ and $T^{c}$ are the colour group generators in the fundamental and adjoint representations respectively.

The second complication is that in the NLO the Reggeon differs essentially from the gluon. Therefore, unlike usual QCD vertices (such as, for example, the quark-quarkgluon vertex) for which one can draw a definite set of Feynman diagrams with perfectly defined rules for calculation of their contributions, we have not such rules for the Reggeon vertices. Usually these vertices are extracted from a comparison of appropriate scattering amplitudes with their Reggeized form, so that to obtain a NLO vertex one has to calculate radiative corrections to a whole amplitude. Nevertheless, it was shown in Refs. [25], [27], that it is possible to formulate definite rules for a calculation of the vertices themselves. In the NLO any vertex can be presented as the sum of two contributions, the first of which is equal to $\Gamma^{(0)} \omega^{(1)}\left(q^{2}\right)\left(\ln \beta_{0}+k / 2\right)$ where $\Gamma^{(0)}$ is the $\mathrm{LO}$ vertex, $\beta_{0}$ is an intermediate parameter for separation of two kinematical regions, $k$ is the process independent constant

$$
k=\frac{1}{\epsilon}+2 \psi(1+2 \epsilon)-2 \psi(1+\epsilon)+\psi(1-\epsilon)-\psi(1)+\frac{5+3 \epsilon-2(1+\epsilon) n_{f} / N_{c}}{2(1+2 \epsilon)(3+2 \epsilon)},
$$

and the second contribution can be obtained as usual one-loop QCD amplitude with ordinary representation in terms of Feynman diagrams, where for the Reggeized gluon interactions one should use the vertices of Fig. 1.

Self-energy insertions in the Reggeon line must be omitted and the Feynman gauge for virtual gluons is assumed. In (2.6) $\psi(z)=\Gamma^{\prime}(z) / \Gamma(z), n_{f}$ is the number of quark flavours. The intermediate parameter $\beta_{0} \rightarrow 0$ (remind, however, that first of all the limit $s \rightarrow \infty$ must be taken) and the dependence on $\beta_{0}$ vanishes in this limit due to the cancellation between the two contributions. The amplitude $\Gamma_{\gamma^{*} R \rightarrow q \bar{q} g}^{c}$ is needed at the Born level only. It can be calculated using the same Feynman rules (the vertices of Fig. 1 and the Feynman gauge for virtual gluons propagators). Generally speaking, for the case of real emission the theta function in the vertex of Fig. 1 should be absent. But for the real emission the limitation on the invariant mass of the $q \bar{q} g$ system in (2.4) leads to

$$
\left|\frac{p_{2}\left(k_{1}-k_{2}\right)}{s}\right| \geq \frac{\vec{k}^{2}}{M^{2}}
$$


where $\vec{k}$ is the transverse momentum of the emitted gluon. We will choose the parameter $\beta_{0} \rightarrow 0$ so small that in the region (2.7) the theta function of Fig. 1 is equal 1 , so that it can be formally written. The amplitude obtained in such a way is gauge invariant with respect to the emitted gluon, that allows to use the Feynman gauge for summation over its polarizations as well.

Therefore we can present the NLO virtual photon impact factor in the form

$$
\begin{gathered}
\Phi_{\gamma^{*}}\left(\vec{q}, s_{0}\right)=\Phi_{M}(\vec{q})-\frac{g^{2} N_{c} \vec{q}^{2}}{(2 \pi)^{D-1}} \int \frac{d^{D-2} q_{1}}{\vec{q}_{1}^{2}\left(\vec{q}_{1}-\vec{q}\right)^{2}} \Phi_{\gamma^{*}}^{(0)}\left(\vec{q}_{1}\right) \ln \frac{M^{4}}{s_{0}\left(\vec{q}_{1}-\vec{q}\right)^{2}} \\
+\omega^{(1)}\left(q^{2}\right) \Phi_{\gamma^{*}}^{(0)}(\vec{q})\left(\ln \frac{s_{0} \beta_{0}^{2}}{\vec{q}^{2}}+k\right),
\end{gathered}
$$

where $k$ is defined in Eq. (2.6) and $\Phi_{M}$ is expressed in terms of the $\tilde{s}$-channel discontinuity of the forward $\gamma^{*} R$ scattering amplitude:

$$
\Phi_{M}(\vec{q})=-2 i \int_{0}^{M^{2}} \frac{d \tilde{s}}{2 \pi} \sum_{n} D_{n}^{(c u t)}(q)=-2 i \int_{0}^{M^{2}} \frac{d \tilde{s}}{2 \pi} \Delta_{\tilde{s}} \sum_{l} D_{l}(q) .
$$

Here $D_{l}$ is the contribution of $l$-th diagram to the $\gamma^{*} R \rightarrow \gamma^{*} R$ amplitude, $\Delta_{\tilde{s}} D_{l}$ is its $\tilde{s}$ - channel discontinuity and $D_{n}^{(c u t)}$ is the contribution to the discontinuity of $n$-th cut diagram calculated according to Cutcosky rule $\left(-2 \pi i \delta_{+}\left(k^{2}\right)\right.$ instead of $1 /\left(k^{2}+i 0\right)$ for a cut line with momentum $k$ ). The factor 2 in (2.9) appears since each diagram has its partner with opposite direction of quark line, which gives the same contribution, and which is not included in the sums in (2.9). There are $34 \mathrm{LO}$ and NLO diagrams for $\gamma^{*} R$ scattering having a discontinuity in the $\tilde{s}$-channel, and 56 cut diagrams (some of diagrams have more than one Cutcosky cuts) in the relation (2.9) for the contribution $\Phi_{M}(\vec{q})$ to the virtual photon impact factor, which we will be mostly concentrated on in the following. We do not present all of them here, but explain our method how to reduce the number of diagrams to be calculated on an example of the simple subset of diagrams shown in Fig. 2. In spite of the simplicity it contains all the relevant to our discussion features of the complete set of diagrams contributing to Eq. (2.9).

First we notice that the diagrams $(a)$ and $(c)$ in Fig. 2, as well as the diagrams $(b)$ and $(d)$ there, differ by the directions of all external lines only. The replacement $c \leftrightarrow c^{\prime}$ does not change anything because of the colour singlet in the $t$ - channel. This means that we may consider, for instance, the first two diagrams of Fig. 2 only, assuming for them

$$
e_{\mu} e_{\nu}^{\prime *} \rightarrow e_{\mu} e_{\nu}^{*}+e_{\mu}^{\prime *} e_{\nu}
$$

with $e$ and $e^{\prime}$ being the polarizations vectors of the incoming and outgoing virtual photons correspondingly. Each diagram in Eq. (2.9) either has such a partner or it is self-symmetric under the symmetrization of the initial and final virtual photons polarizations. Further we always assume the prescription (2.10) for each diagram we work with, that reduces the number of Feynman diagrams to be considered in the relation (2.9) to 22, 2 (LO) and 20 (NLO) shown in Figs. 4, 5(a) and Fig. 7. In the next sections we will show how to reduce more this number using analytical properties of the diagrams. 


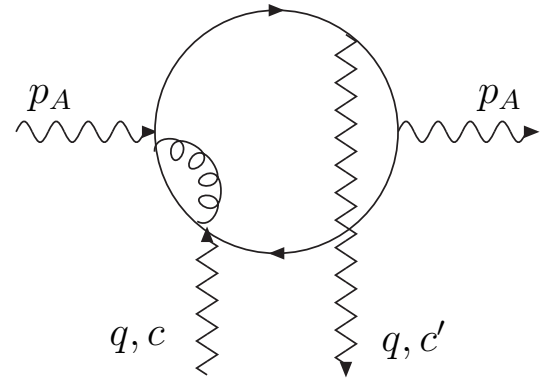

(a)

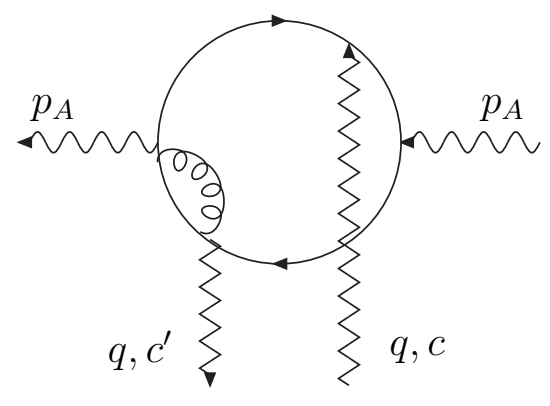

(c)

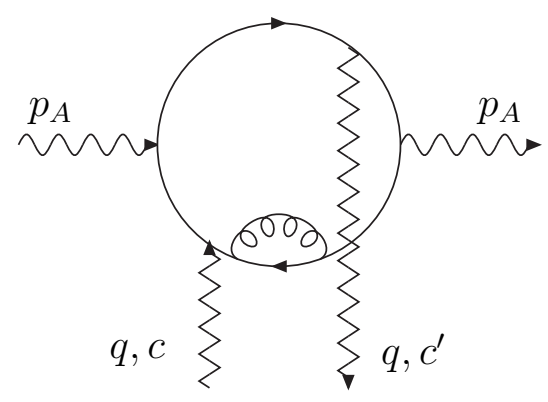

(b)

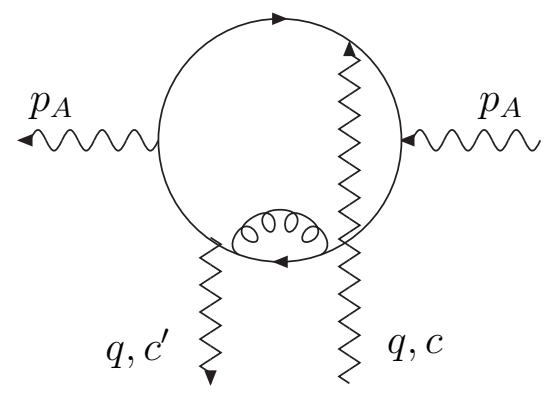

$(d)$

Figure 2: Some of diagrams contributing to Eq. (2.9).

\section{Analytical properties of $D_{n}$}

Let us consider one of the contributions $D_{n}$ in Eq. (2.9). It is an analytical function of variable $\tilde{s}$ having a branch cut discontinuity at $0<\tilde{s}<\infty$ and, possibly, also at $-\infty<\tilde{s}<-2\left(\vec{q}^{2}+Q^{2}\right)$ related to the $\tilde{u}$ - channel Cutcosky cuts with

$$
\tilde{u}=\left(p_{A}-q\right)^{2}=-2\left(\vec{q}^{2}+Q^{2}\right)-\tilde{s} .
$$

We need the integral (see Fig. 3)

$$
\int_{0}^{M^{2}} d \tilde{s} \Delta_{\tilde{s}} D_{n}(q)=\int_{C_{+}} d \tilde{s} D_{n}(q)=-\int_{C_{M}} d \tilde{s} D_{n}(q)-\int_{C_{-}} d \tilde{s} D_{n}(q) .
$$

Using Eqs. (2.1), (3.1), the last integral here can be presented as

$$
-\int_{C_{-}} d \tilde{s} D_{n}(q)=\int_{0}^{M^{2}} d \tilde{u} \Delta_{\tilde{u}} D_{n}(q)=\int_{0}^{M^{2}} d \tilde{s} \Delta_{\tilde{s}} D_{n}\left(-q^{\prime}\right)=\int_{0}^{M^{2}} d \tilde{s} \Delta_{\tilde{s}} \tilde{D}_{n}\left(q^{\prime}\right),
$$

where

$$
q^{\prime}=\frac{\tilde{s}+Q^{2}+\vec{q}^{2}}{s} p_{2}-q_{\perp}=\left.q\right|_{q_{\perp} \leftrightarrow-q_{\perp}},
$$




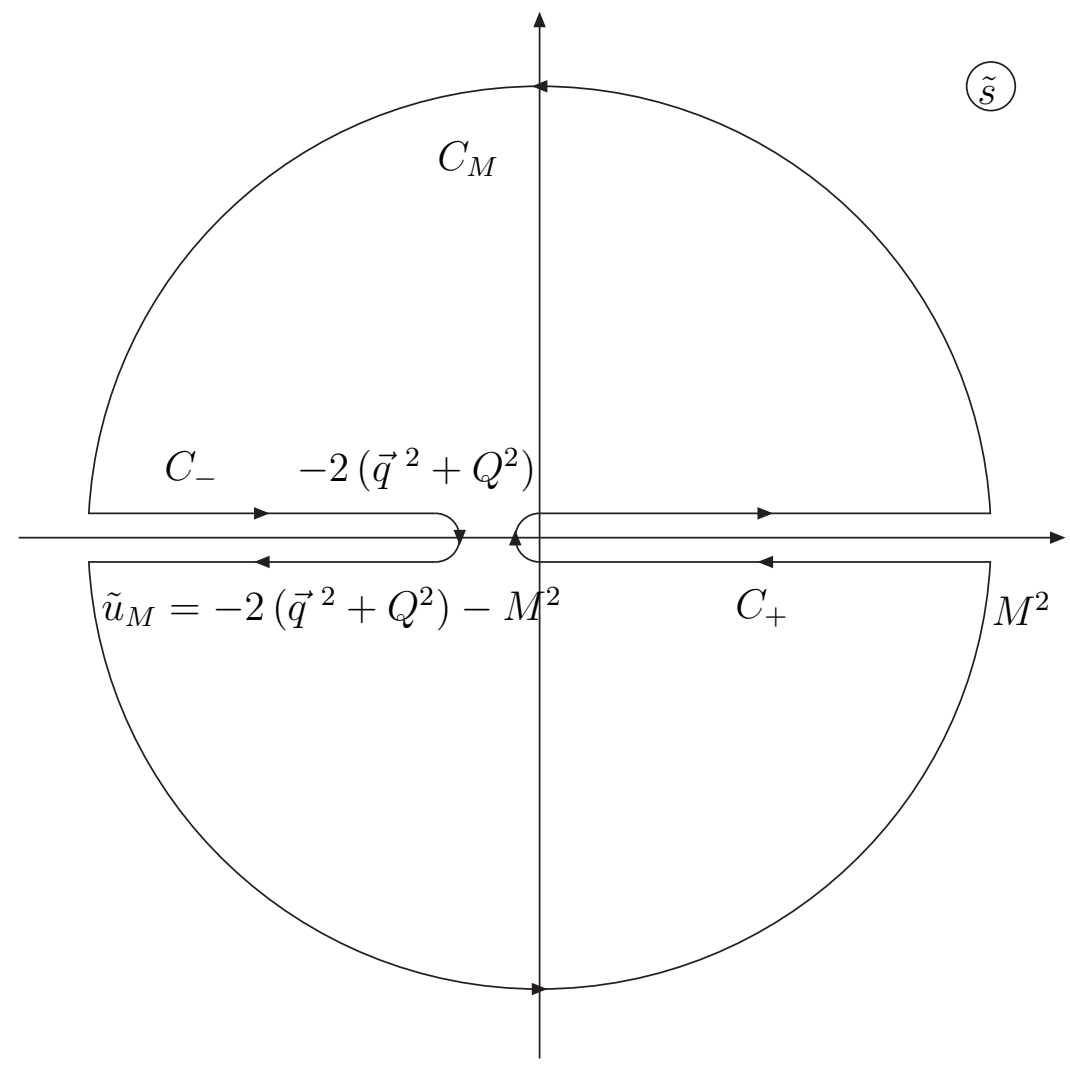

Figure 3: Schematic representation of analytical properties of $D_{n}$.

and $\tilde{D}_{n}$ differs from $D_{n}$ by the directions of Reggeon lines only. In the light-cone gauge

$$
e p_{2}=e^{\prime} p_{2}=0
$$

which we use, the contribution of each diagram can be decomposed over four independent spin structures which we define as follows

$$
\begin{gathered}
T^{(+)}=\frac{-e_{\mu} e_{\nu}^{*}}{1+\epsilon} g_{\perp}^{\mu \nu}, \quad T^{(-)}=\frac{-e_{\mu} e_{\nu}^{* *}}{(1+\epsilon)(1+2 \epsilon)}\left(g_{\perp}^{\mu \nu}-(D-2) \frac{q_{\perp}^{\mu} q_{\perp}^{\nu}}{q_{\perp}^{2}}\right) \\
L^{(+)}=e_{\mu} e_{\nu}^{\prime *} \frac{p_{A}^{\mu} p_{A}^{\nu}}{Q^{2}}, \quad L^{(-)}=\left(e_{\mu} e_{\nu}^{* *}+e_{\mu}^{\prime *} e_{\nu}\right) \frac{2 p_{A}^{\mu} q_{\perp}^{\nu}}{\sqrt{2 \vec{q}^{2} Q^{2}}}
\end{gathered}
$$

where

$$
g_{\perp}^{\mu \nu}=g^{\mu \nu}-\frac{p_{1}^{\mu} p_{2}^{\nu}+p_{2}^{\mu} p_{1}^{\nu}}{p_{1} p_{2}} .
$$

The spin structures $T^{(+)}$and $L^{(+)}$describe the transverse and the longitudinal spin-nonflip transitions respectively, the other two structures, $T^{(-)}$and $L^{(-)}$, correspond to the double and the single spin-flip helicity amplitudes. Therefore we come to the conclusion

$$
\tilde{D}_{n}\left(q^{\prime}\right)=\left.\tilde{D}_{n}(q)\right|_{q_{\perp} \leftrightarrow-q_{\perp}}=\left.\tilde{D}_{n}(q)\right|_{L^{(-)} \leftrightarrow-L^{(-)}},
$$


and finally, using Eqs. (3.2), (3.3) and (3.8), we get

$$
\int_{0}^{M^{2}} d \tilde{s} \Delta_{\tilde{s}} D_{n}(q)=-\int_{C_{M}} d \tilde{s} D_{n}(q)+\left.\int_{0}^{M^{2}} d \tilde{s} \Delta_{\tilde{s}} \tilde{D}_{n}(q)\right|_{L^{(-)} \leftrightarrow-L^{(-)}} .
$$

Eq. (3.9) can be written for any diagram in (2.9); for those of them without $\tilde{u}$ - channel cut the last term in (3.9) does not appear. Let us consider the application of the relation (3.9) for the diagrams in Fig. 2. According to the discussion above, their contribution to $\Phi_{M}$ takes the form

$$
\begin{gathered}
\Phi_{M}^{(\text {Fig. 2) }}=-2 i \int_{0}^{M^{2}} \frac{d \tilde{s}}{2 \pi} \Delta_{\tilde{s}}\left(D_{2(a)}+D_{2(b)}\right) \\
=2 i \int_{C_{M}} \frac{d \tilde{s}}{2 \pi} D_{2(b)}-2 i \int_{0}^{M^{2}} \frac{d \tilde{s}}{2 \pi} \Delta_{\tilde{s}}\left(D_{2(a)}+\left.\tilde{D}_{2(b)}\right|_{L^{(-)} \leftrightarrow-L^{(-)}}\right),
\end{gathered}
$$

where we have applied (3.9) to the diagram $D_{2(b)}$. It is easy to see that

$$
\tilde{D}_{2(b)}=D_{2(a)}
$$

therefore

$$
\Phi_{M}^{(F i g .2)}=2 i \int_{C_{M}} \frac{d \tilde{s}}{2 \pi} D_{2(b)}-\left.4 i \int_{0}^{M^{2}} \frac{d \tilde{s}}{2 \pi} \Delta_{\tilde{s}} D_{2(a)}\right|_{L^{(-)}=0}
$$

Comparing Eqs. (3.10) and (3.12), we see, that if we worked in a scalar quantum field theory (let us say $\phi^{3}$ for definiteness) we could consider only one diagram instead of two, because the contribution of the large circle would disappear due to fast enough decrease of amplitudes at large $\tilde{s}$. The situation in QCD is more complicated: contributions from the integration over the large circle basically survive for separate diagrams. Nevertheless, we will show in the following that analyticity helps to consider many diagrams without their real calculation in the QCD case also.

After a short consideration it becomes clear that the transformations we used to obtain (3.12), the result for the contribution of the set of diagrams shown in Fig. 2, may be applied as well to the complete set of 22 diagrams which contribute to Eq. (2.9). Proceeding along the same steps we arrive at the following representation of the impact factor

$$
\Phi_{M}=\Phi_{\Delta}+\Phi_{\Lambda}
$$

where the first term is given by the integral over $\tilde{s}$ - channel discontinuity of 12 diagrams of Fig. 4,

$$
\begin{gathered}
\Phi_{\Delta}=-i \int_{0}^{M^{2}} \frac{d \tilde{s}}{2 \pi} \Delta_{\tilde{s}}\left[D_{4(a)}+\cdots+D_{4(c)}\right. \\
\left.+2\left(D_{4(d)}+\cdots+D_{4(j)}\right)+\left.2\left(D_{4(k)}+2 D_{4(l)}\right)\right|_{L^{(-)}=0}\right]
\end{gathered}
$$

whereas the second contribution, $\Phi_{\Lambda}$, consists of the integrals over the large circle from 10 diagrams shown in Fig. 5(a) and in Fig. 7. To see this one needs to move the integral over the $\tilde{s}$ - channel discontinuity for these diagrams to the integral over the infinite circle. 


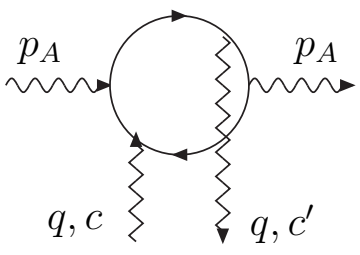

(a)

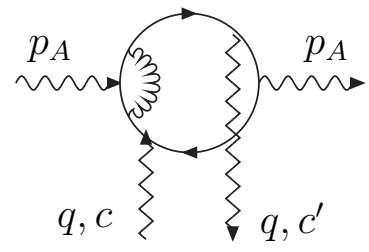

(d)

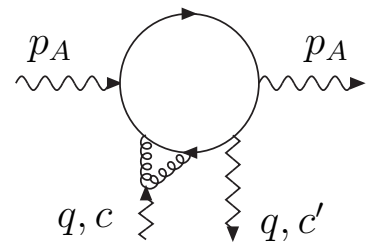

$(g)$

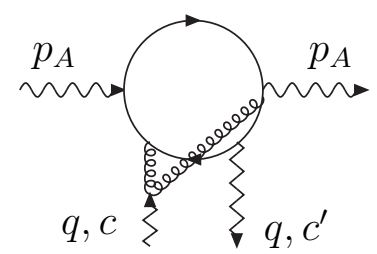

$(j)$

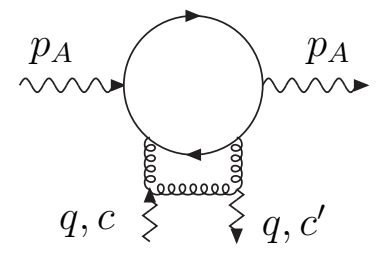

(b)

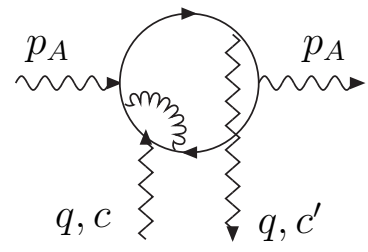

(e)

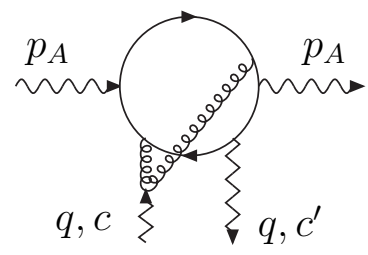

(h)

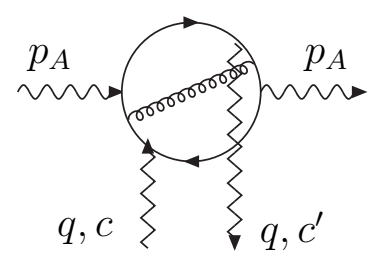

$(k)$

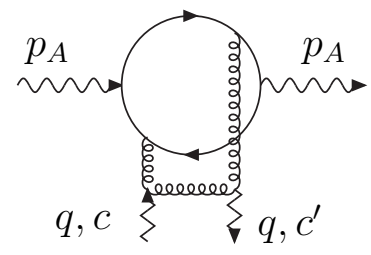

(c)

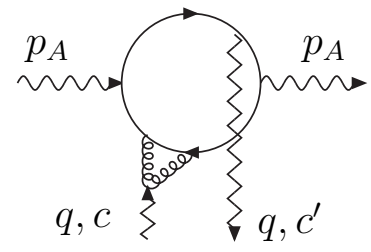

$(f)$

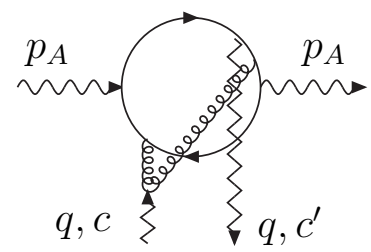

(i)

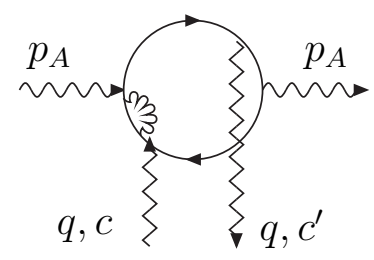

(l)

Figure 4: The diagrams contributing to $\Phi_{\Delta}$.

Two of the diagrams in Fig. 7, $D_{2}^{r}$ and $D_{3}^{r}$, have both $\tilde{s}$ - and $\tilde{u}$ - channel singularities. As it was demonstrated above the contributions of the $\tilde{u}$ - channel cuts for these diagrams may be related to the integrals over $\tilde{s}$ - channel discontinuities of their symmetric partners, diagrams $(l)$ and $(k)$ in Fig. 4. That is quite natural since the $\tilde{s}$ - and $\tilde{u}$ - channels are actually the same in the case under consideration. We used this symmetry deriving our representation for the impact factor, this is why factor 2 is present in the last term of Eq. (3.14). The discontinuity $\Delta_{\tilde{s}}$ in this relation is given by the $\tilde{s}$ - channel Cutcosky cuts. There are 18 cut diagrams corresponding to the diagrams of Fig. 4.

In the next section we will concentrate on the contribution $\Phi_{\Lambda}$ in Eq. (3.13) given by the integrals over the large circle. We will prove that $\Phi_{\Lambda}$ does not depend on the Reggeon transverse momentum $\vec{q}$. That makes it possible to express $\Phi_{M}(\vec{q})$ through $\Phi_{\Delta}$ (3.14) only, and to avoid, therefore, an explicit calculation of $\Phi_{\Lambda}$. 


\section{Large circle contribution}

In order to consider the contribution $\Phi_{\Lambda}$ we introduce new longitudinal subspace basis $\left(p_{1}, p_{2}^{\prime}\right)$ with

$$
p_{2}^{\prime}=\frac{\tilde{s}+Q^{2}+\vec{q}^{2}}{s} p_{2}, \quad 2 p_{1} p_{2}^{\prime}=s_{1}=\tilde{s}+Q^{2}+\vec{q}^{2}=\frac{\tilde{s}-\tilde{u}}{2}
$$

Therefore

$$
q=p_{2}^{\prime}+q_{\perp}, \quad p_{A}=p_{1}-\frac{Q^{2}}{s_{1}} p_{2}^{\prime},
$$

and the gauge fixing condition for the external virtual photons remains actually the same (compare with the Eq.(3.5))

$$
e p_{2}^{\prime}=e^{\prime} p_{2}^{\prime}=0
$$

Let us consider first the Born contribution to $\Phi_{\Lambda}$

$$
\Phi_{\Lambda}^{(0)}=-i\left(e q_{f} g\right)^{2} \frac{\sqrt{N_{c}^{2}-1}}{4 \pi} \int_{C_{M}} \frac{d \tilde{s}}{s_{1}^{2}} D_{a}^{r},
$$

where $e q_{f}$ is the quark electric charge and $D_{a}^{r}$ is the amplitude corresponding to the diagram of Fig. 5(a), calculated with the following changes with respect to the Feynman rules used before (we adopt these changes everywhere in this section)

1. $-i g t^{a}$ - factors are removed from all QCD vertices as well as $-i e q_{f} I$ from QED ones so that nothing except corresponding Dirac $\gamma$ - matrix remains in any vertex;

2. $i I$ from quark propagators and $-i \delta^{a b}$ from gluon ones (they do not appear in $D_{a}^{r}$ but they are present in other diagrams for $\left.\Phi_{\Lambda}\right)$ are also removed as it is shown in Fig. 5(b);

3. the factor -1 corresponding to the quark loop is omitted;

4. in quark-Reggeon vertices Reggeons are replaced by gluons with polarization vectors equal $p_{2}^{\prime}$ (as it is explicitly indicated in the Fig. 5(a)), since the Reggeon interacts with quarks exactly as the gluon with such polarization. Remind that for photon polarizations the substitution (2.10) is assumed.

Using the relation (4.2) together with the above agreement for the Feynman rules, the amplitude $D_{a}^{r}$ can be decomposed as it is shown at Fig. 5(c). Then we notice that the last term in Fig. 5(c) with external gluon polarizations $q_{\perp}$ can be omitted since it does not generate a growing with $\tilde{s}$ contribution required by Eq. (4.4). This is because to obtain the growing contribution at limited transverse polarization vectors of the external gluons one needs purely gluonic intermediate states in the $t$ - channel. So, $D_{a}^{r}$ can be presented by the last but one term in the graphic relation of Fig. 5(c). To investigate further its high energy behaviour we apply the Ward identities shown in a graphic form at Fig. $5(\mathrm{~d})$. They allow to present $D_{a}^{r}$ in the form given by the first equality of Fig. 6(a). 


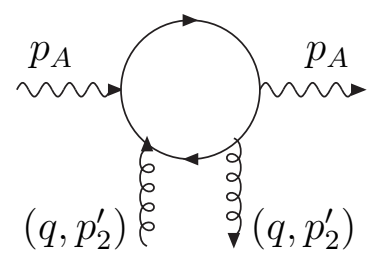

(a)

$$
\stackrel{k}{\longrightarrow}=k^{-1}, \stackrel{\mu}{\mu} \stackrel{\text { mor }}{\nu}=g_{\mu \nu} /\left(k^{2}+i \delta\right) .
$$

(b)
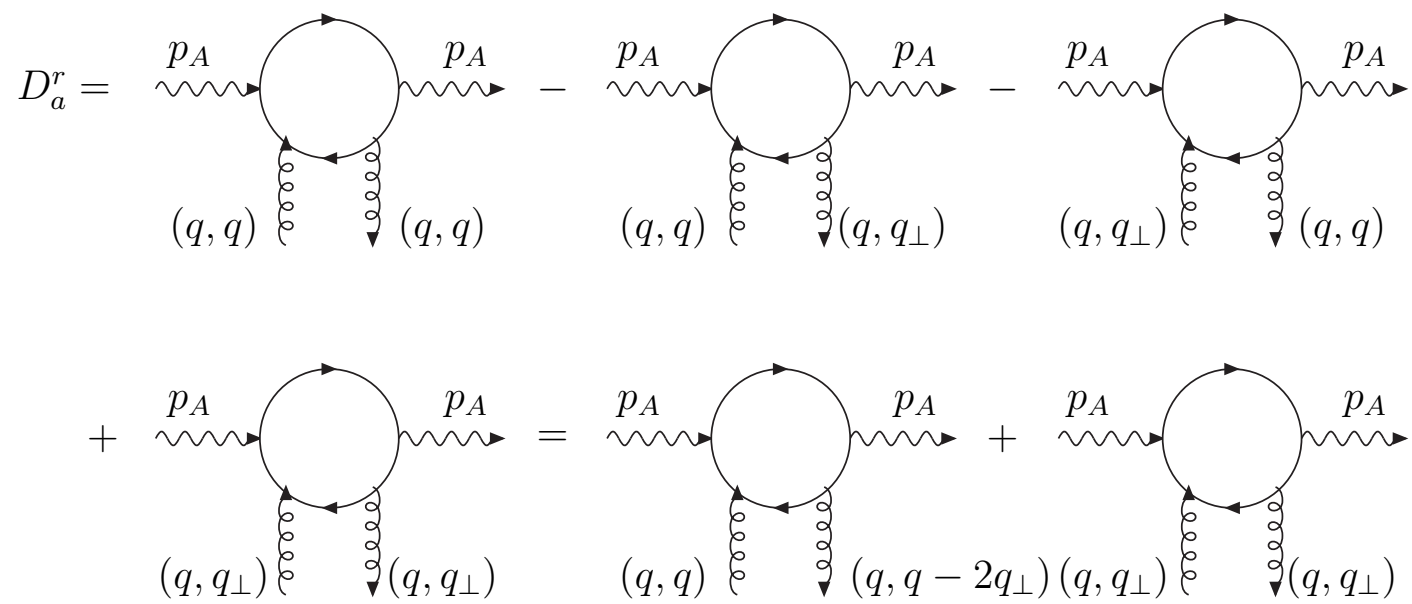

(c)

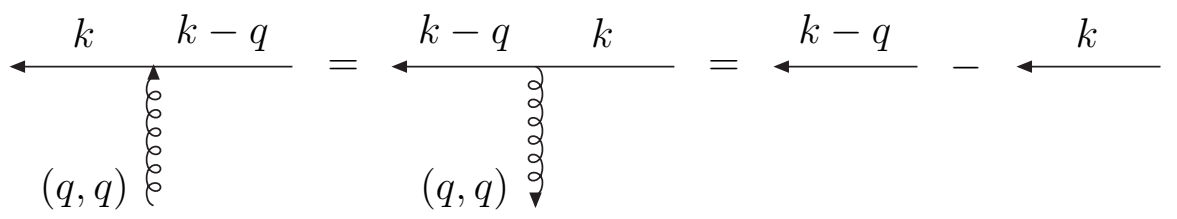

$(d)$

Figure 5: a) the diagram contributing to $\Phi_{\Lambda}^{(0)}$; b) prescriptions for quark and gluon lines used in Section 4; c) graphic representation of the decompositions of $D_{a}^{r}$; d) the Ward identities in a graphic form.

We then notice that the parts of the diagrams in this equality related to the $q_{\perp^{-}}$ polarizations can be omitted because the corresponding contributions do not grow with $\tilde{s}$. This admits to apply again the Ward identities and obtain the second equality at Fig. 6(a), where the last diagram evidently does not grow with $\tilde{s}$ and can be omitted.

As for the last but one diagram of Fig. 6(a) we note, first of all, that the external virtual photons there have different from $p_{A}$ momenta (as it is indicated explicitly), so that the corresponding amplitude can grow with $\tilde{s}$. Nevertheless, it can be omitted also. The reason is that the only energy scale for this amplitude is just $\tilde{s}$ and therefore its high energy behaviour in the used by us dimensional regularization is fixed to be $(-\tilde{s})^{\epsilon+1}$, so that its contribution to (4.4) is proportional to the following expression

$$
\int_{C_{M}} \frac{d \tilde{s}}{2 \pi i}(-\tilde{s})^{\epsilon-1}=-\int_{0}^{M^{2} \rightarrow \infty} \frac{d \tilde{s}}{2 \pi i} \Delta_{\tilde{s}}(-\tilde{s})^{\epsilon-1}=-\frac{\sin (\pi \epsilon)}{\pi} \int_{0}^{\infty} d \tilde{s}(\tilde{s})^{\epsilon-1}=0 .
$$

It vanishes since we first tend $M$ to infinity and only after that $\epsilon$ goes to 0 , as it is 

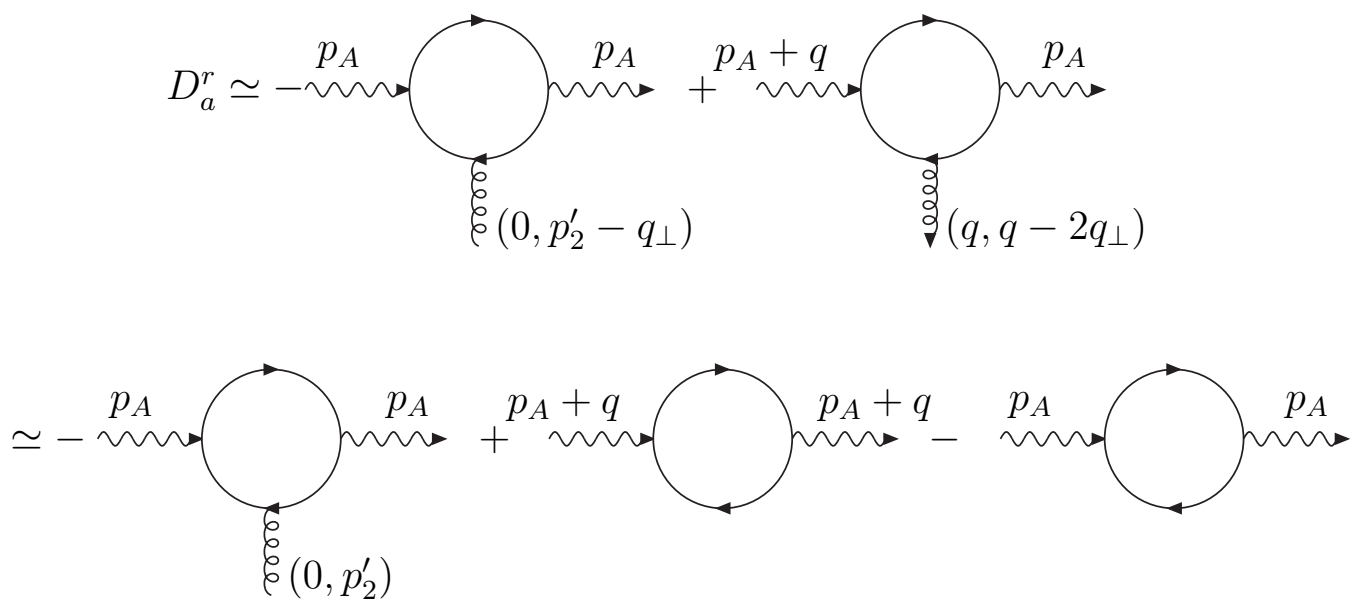

(a)
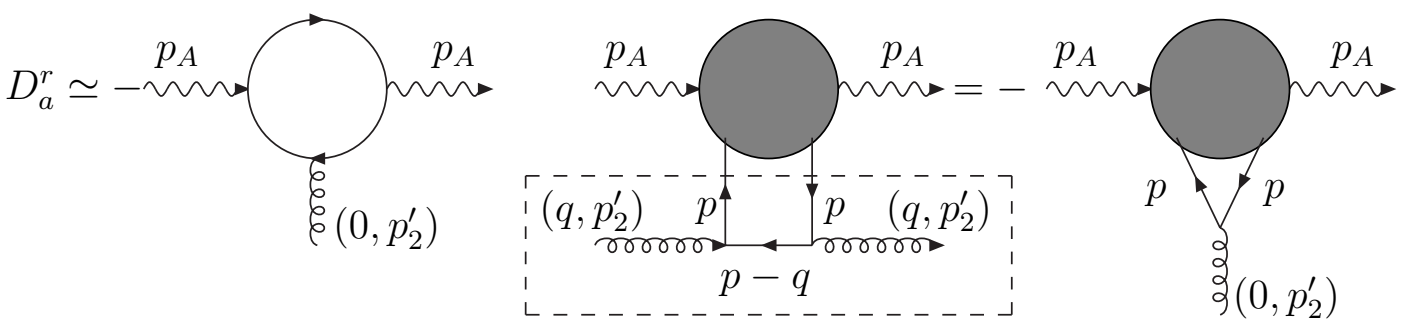

(b)

(c)

Figure 6: a) decompositions of $D_{a}^{r}$ with the use of the Ward identities; b) the final result for the high energy asymptotics of $D_{a}^{r}$; c) the illustration of this final result.

done systematically in the BFKL approach (see Refs. [6] - [10], for instance). Of course, these two limits must commute in final infrared stable results for observables, but at intermediate steps the order of the limits adopted from the beginning must be kept the same everywhere.

We come therefore to the result for the high energy asymptotics of $D_{a}^{r}$ shown graphically in Fig. 6(b). This result demonstrates the complete independence of $D_{a}^{r}$ from the Reggeon transverse momentum $q_{\perp}$. This means the independence from $q_{\perp}$ also for $\Phi_{\Lambda}^{(0)}$ itself, and, as we will shortly see, this property is valid for the complete NLO $\Phi_{\Lambda}$. Here we make one more remark which will be used in the following: the diagram for $D_{a}^{r}$ has a form of the diagram of Fig. 6(c) where the marked by a dash box piece can be written down as follows

$$
\frac{\not p_{2}^{\prime}(\not p-\not q) \not p_{2}^{\prime}}{(p-q)^{2}+i \delta}=-\not p_{2}^{\prime}+\not p_{2}^{\prime} \frac{(\vec{p}-\vec{q})^{2}-\alpha \beta s_{1}}{(1-\alpha) \beta s_{1}+(\vec{p}-\vec{q})^{2}-i \delta},
$$

in the Sudakov variables

$$
p=\beta p_{1}+\alpha p_{2}^{\prime}+p_{\perp} .
$$

At limited transverse momentum

$$
\vec{p}^{2} \sim \vec{q}^{2} \sim Q^{2},
$$




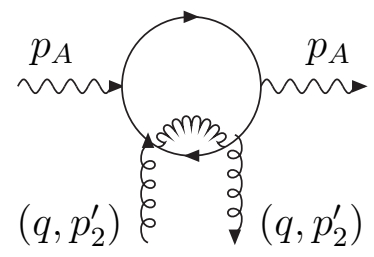

$\left(D_{1}^{r}\right)$

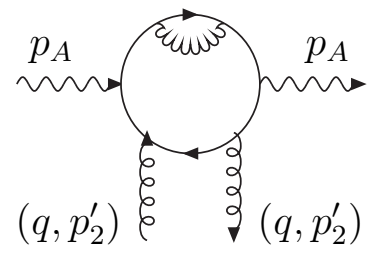

$\left(D_{4}^{r}\right)$

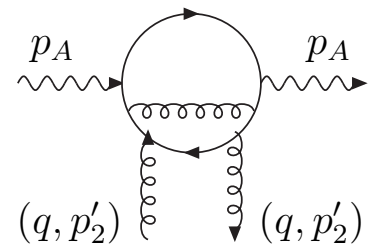

$\left(D_{7}^{r}\right)$

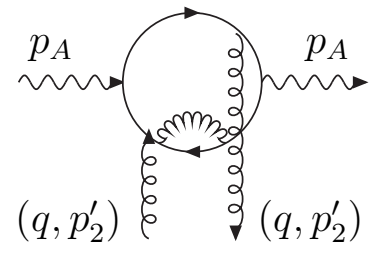

$\left(D_{2}^{r}\right)$

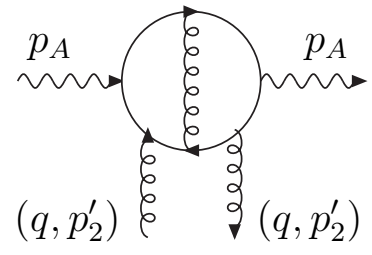

$\left(D_{5}^{r}\right)$

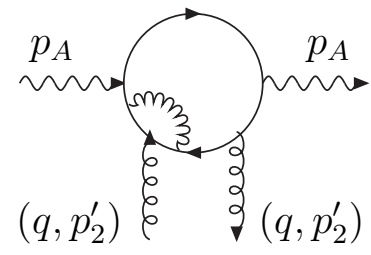

$\left(D_{8}^{r}\right)$

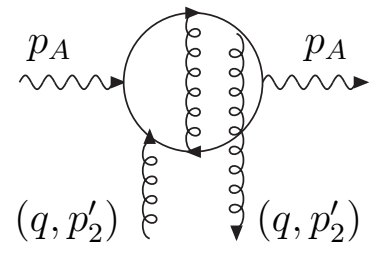

$\left(D_{3}^{r}\right)$
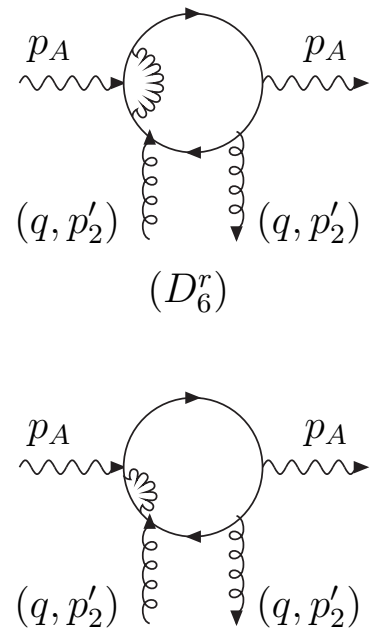

$\left(D_{9}^{r}\right)$

Figure 7: The diagrams contributing to $\Phi_{\Lambda}^{(1)}$.

the essential integration region in the longitudinal subspace is

$$
\alpha \sim \vec{q}^{2} / s_{1} \ll 1, \quad \beta \sim 1 .
$$

In this region the last term of Eq. (4.6) is suppressed and can be omitted so that the expression inside the dash box of Fig. 6(c) becomes just

$$
\frac{\not p_{2}^{\prime}(\not p-\not q) \not p_{2}^{\prime}}{(p-q)^{2}+i \delta} \rightarrow-\not p_{2}^{\prime} .
$$

As for the region of integration over large transverse momentum, it always brings the energy dependence like that of the Eq. (4.5) and can be also neglected. The prescription (4.10) provides the relation depicted schematically in Fig. 6(c) (of course, it reproduces the correct result for $D_{a}^{r}$ ).

We now turn to consideration of the complete $\mathrm{NLO} \Phi_{\Lambda}$ which can be written as the sum of three contributions

$$
\Phi_{\Lambda}=\Phi_{\Lambda}^{(0)}+\Phi_{\Lambda}^{(1)(a)}+\Phi_{\Lambda}^{(1)(n a)},
$$

where the LO part $\Phi_{\Lambda}^{(0)}$ is given by (4.4) and the NLO parts are expressed in terms of 9 diagrams of Fig. 7 


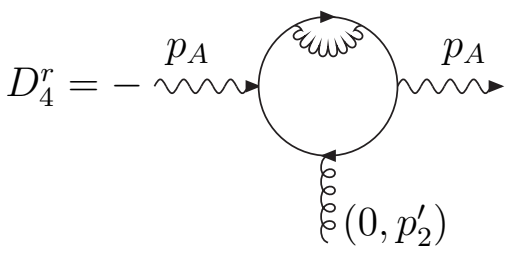

(a)

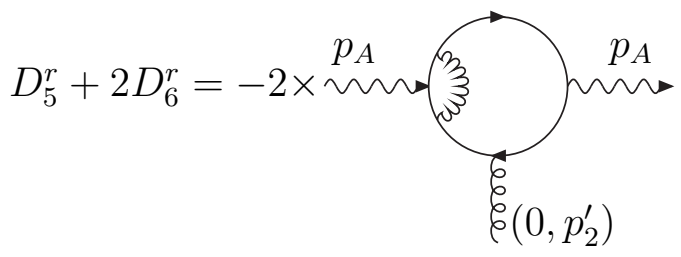

(b)

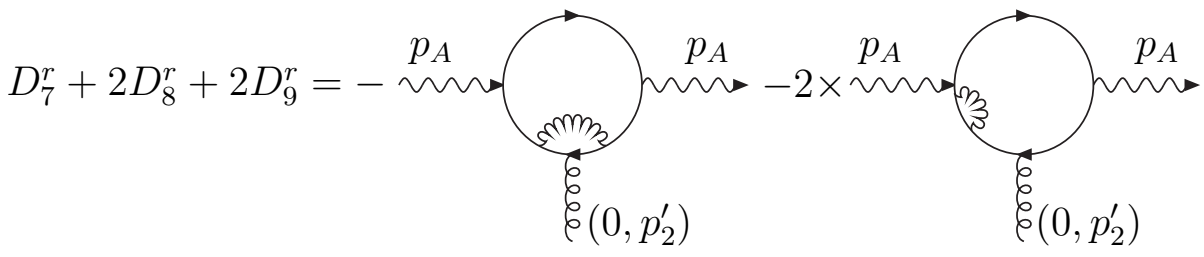

(c)

Figure 8: Graphic relations for the diagrams of the Fig. 7.

$$
\begin{gathered}
\Phi_{\Lambda}^{(1)(a)}=-\left(e q_{f} g^{2}\right)^{2} \frac{C_{F} \sqrt{N_{c}^{2}-1}}{4 \pi} \\
\times \int_{C_{M}} \frac{d \tilde{s}}{s_{1}^{2}}\left[D_{1}^{r}+2 D_{2}^{r}+D_{3}^{r}+D_{4}^{r}+D_{5}^{r}+2 D_{6}^{r}+D_{7}^{r}+2\left(D_{8}^{r}+D_{9}^{r}\right)\right], \\
\Phi_{\Lambda}^{(1)(n a)}=\left(e q_{f} g^{2}\right)^{2} \frac{C_{A} \sqrt{N_{c}^{2}-1}}{8 \pi} \int_{C_{M}} \frac{d \tilde{s}}{s_{1}^{2}}\left[D_{3}^{r}+D_{5}^{r}+2 D_{8}^{r}\right],
\end{gathered}
$$

with the standard notations $C_{F}$ and $C_{A}$ for the Casimir operators in the fundamental and adjoint $S U\left(N_{c}\right)$ colour group representations respectively. For the first three diagrams of Fig. 7 the region of integration over limited transverse momenta does not give a growing with $\tilde{s}$ contribution so that they separately can be omitted due to relations similar to (4.5):

$$
\int_{C_{M}} \frac{d \tilde{s}}{s_{1}^{2}} D_{1}^{r}=\int_{C_{M}} \frac{d \tilde{s}}{s_{1}^{2}} D_{2}^{r}=\int_{C_{M}} \frac{d \tilde{s}}{s_{1}^{2}} D_{3}^{r}=0 .
$$

For other diagrams of Fig. 7, using the Ward identities, one can obtain the relations presented in graphic form by Fig. 8 .

Let us note that the diagrams for $D_{4}^{r}, D_{6}^{r}, D_{7}^{r}$ and $D_{9}^{r}$ are of the kind of Fig. 6(c) and satisfy therefore the same relation in their high energy limit. It means that $2 D_{6}^{r}$ and $D_{7}^{r}+2 D_{9}^{r}$ saturate the equalities of Figs. 8(b) and (c) correspondingly, so that $D_{5}^{r}$ and $D_{8}^{r}$ disappear (separately), although it is not so evident as in the case of the first three diagrams of Fig. 7.

The final conclusion of this Section is that the NLO $\Phi_{\Lambda}$ consists of the integrals over the large circle in the complex $\tilde{s}$ - plane from the amplitudes corresponding to the diagrams presented by Figs. 5(a) and 7, which either have the structure of the Fig. 6(c) and do not therefore depend on $q_{\perp}$ in the high energy limit or separately disappear in this limit. It leads to the independence from the Reggeon transverse momentum also for the $\Phi_{\Lambda}$ itself. This conclusion is valid in the Feynman gauge for virtual gluons which we use here. 


\section{Conclusions}

The virtual photon impact factor $\Phi_{\gamma^{*}}\left(\vec{q}, s_{0}\right)$, being the impact factor of the colourless object, has an important property

$$
\Phi_{\gamma^{*}}\left(\vec{q}=0, s_{0}\right)=0
$$

that is related to the gauge invariance and is necessary for the infrared finiteness of the cross section describing the collision of colourless particles. Since the last two terms in (2.8) vanish at $\vec{q}=0$, the contribution $\Phi_{M}(\vec{q})$ has also to possess this property

$$
\Phi_{M}(\vec{q}=0)=\Phi_{\Delta}(\vec{q}=0)+\Phi_{\Lambda}(\vec{q}=0)=0 .
$$

We have shown in the previous Section that $\Phi_{\Lambda}$ does not depend on $\vec{q}$. It means that $\Phi_{\Lambda}(q)=-\Phi_{\Delta}(q=0)$ and $\Phi_{M}(\vec{q})$ can be presented as

$$
\Phi_{M}(\vec{q})=\Phi_{\Delta}(\vec{q})-\Phi_{\Delta}(\vec{q}=0),
$$

where $\Phi_{\Delta}$ defined by the Eq. (3.14) and Fig. 4 .

We came to the above simplified representation using the analytical properties of the Feynman diagrams of the effective quantum field theory with Reggeized gluon included. A part of the diagrams in the expression (2.9) for the impact factor with known high energy behaviour was moved from the integral over the $\tilde{s}$ - channel discontinuity to the integral over the infinite circle in the complex squared energy plane. According to our results this part serves just as a counterterm restoring the correct small $\vec{q}$ behaviour of the other part of the impact factor $\Phi_{\Delta}$, so that it does not have to be calculated explicitly. In other words, we have performed the cancellation of some irrelevant terms before doing the real calculations.

Let us note that the approach using analytical properties of amplitudes of the effective field theory can be useful also for calculation of other NLO impact factors. It is analogous to the approach which was used in QED for the derivation of the sum rules relating the cross sections of various production processes in the Weizsaker-Williams approximation and the slope of the Dirac form factor at zero momentum transfer [28].

To complete our conclusions let us consider how the approach works in the simplest case of the Born virtual photon impact factor. Here we have

$$
\Phi_{\gamma^{*}}^{(0)}(\vec{q})=\Phi_{\Delta}^{(0)}(\vec{q})-\Phi_{\Delta}^{(0)}(\vec{q}=0), \quad \Phi_{\Delta}^{(0)}(\vec{q})=-i \int_{0}^{\infty} \frac{d \tilde{s}}{2 \pi} \Delta_{\tilde{s}} D_{4(a)}
$$

where only the first diagram in Fig. 4 contributes. The calculation becomes very simple and we just quote the result without presentation of any details

$$
\begin{aligned}
\Phi_{\Delta}^{(0)}(\vec{q}) & =e^{2} g^{2} \sqrt{N_{c}^{2}-1} \sum_{f} q_{f}^{2} \frac{\Gamma(1-\epsilon)}{(4 \pi)^{2+\epsilon}}\left\{\left(Q^{2}\right)^{\epsilon} \frac{2}{\epsilon} \int_{0}^{1} d x(x(1-x))^{\epsilon}\right. \\
\times & {\left[(1+\epsilon)(1+\epsilon-2 x(1-x)) T^{(+)}-4 \epsilon x(1-x) L^{(+)}\right] }
\end{aligned}
$$




$$
\begin{gathered}
+\int_{0}^{1} \int_{0}^{1} \frac{d x d y \vec{q}^{2}}{\left(y(1-y) \vec{q}^{2}+x(1-x) Q^{2}\right)^{1-\epsilon}}[(1+\epsilon-2 x(1-x)) \\
\times(1+\epsilon-2(1+2 \epsilon) y(1-y)) T^{(+)}+4 x(1-x)(2(1+2 \epsilon) y(1-y)-\epsilon) L^{(+)} \\
\left.\left.+4(1+2 \epsilon) x(1-x) y(1-y) T^{(-)}\right]\right\} .
\end{gathered}
$$

According to our approach, Eq. (5.4), the LO virtual photon impact factor itself is given by the above expression without the first term in the curly brackets

$$
\begin{gathered}
\Phi_{\gamma^{*}}^{(0)}(\vec{q})=e^{2} g^{2} \sqrt{N_{c}^{2}-1} \sum_{f} q_{f}^{2} \frac{\Gamma(1-\epsilon)}{(4 \pi)^{2+\epsilon}} \int_{0}^{1} \int_{0}^{1} \frac{d x d y \vec{q}^{2}}{\left(y(1-y) \vec{q}^{2}+x(1-x) Q^{2}\right)^{1-\epsilon}}[(1+\epsilon \\
-2 x(1-x))(1+\epsilon-2(1+2 \epsilon) y(1-y)) T^{(+)}+4 x(1-x)(2(1+2 \epsilon) y(1-y)-\epsilon) L^{(+)} \\
\left.+4(1+2 \epsilon) x(1-x) y(1-y) T^{(-)}\right] \approx \sqrt{N_{c}^{2}-1} \sum_{f} q_{f}^{2} \frac{e^{2} g^{2}}{(4 \pi)^{2}} \int_{0}^{1} \int_{0}^{1} \frac{d x d y \vec{q}^{2}}{y(1-y) \vec{q}^{2}+x(1-x) Q^{2}} \\
\times\left[(1-2 x(1-x))(1-2 y(1-y)) T^{(+)}+8 x(1-x) y(1-y)\left(L^{(+)}+\frac{1}{2} T^{(-)}\right)\right],
\end{gathered}
$$

where the last approximate equality shows the result in the physical limit $\epsilon=0$ while the first exact in $\epsilon$ equation is necessary in the NLO to evaluate the counterterm in (2.8). It can be checked by direct calculation that the first term in r.h.s of Eq. (5.5) which we subtracted according to our procedure (5.4) coincides, of course, with the contribution (with the reverse sign) of the diagram Fig. 5(a). So, considering $\Phi_{\gamma^{*}}^{(0)}(\vec{q})$ we need to calculate in a frame of our method only one diagram instead of two.

It should be mentioned that, in a difference to approach used in [19] - [23], the method described above implies an integration at the intermediate stages over the phase space of an individual cut diagram. This integration is divergent in ultraviolet and is regularized in a dimensional regularization method. See, for instance, the first term of Eq. (5.5), where the singularity at $\varepsilon \rightarrow 0$ is an ultraviolet pole. These additional ultraviolet singularities disappear of course in the difference of two terms in Eq. (5.3). At LO this cancellation is very simple and results just in removing the first term in Eq. (5.5) which does not depend on $\vec{q}$. At NLO the situation is more complicated since cut diagrams involve one-loop amplitudes that, in practice, one needs to expand in $\epsilon$ in order to proceed with subsequent calculations. At this stage one has to use with care the results for cut diagrams reported previously in [19] - [23]. In some kinematical regions the procedure of the $\varepsilon$ - expansion for the one-loop amplitudes may need a revision.

Eq. (5.6) restores the well known answer [18]. The calculation in the NLO, instead, remains to be rather complicated even with the use of the simplified expression (5.3). Nevertheless, it is to be done since it has a number of the phenomenological applications. We hope to solve this problem in our subsequent publications.

Acknowledgment: Two of us (M.K. and D.I.) thank the Dipartimento di Fisica della Università della Calabria for the warm hospitality while a part of this work was done. 
This work was partly supported by the INTAS (00-0036 and 00-00679) and by the Russian Fund of Basic Researches (00-15-96691 and 01-02-16042). D.I. was supported by DFG and BMBF (06OR984). V.S.F. thanks the Alexander von Humboldt foundation for the research award, the Universität Hamburg and DESY for their warm hospitality while a part of this work was done.

\section{References}

[1] OPAL Collaboration, G. Abbiendi et al., CERN-EP-2001-06 (2001), hep-ex/0110006.

[2] L3 Collaboration, P. Achard et al., CERN-EP-2001-075 (2001), hep-ex/0111012.

[3] Aleph Collaboration, presented by G. Prange at PHOTONS-2001, Ascona, Switzerland, September 2-7, 2001.

[4] V.S. Fadin, E.A. Kuraev, L.N. Lipatov, Phys. Lett. B60 (1975) 50; E.A. Kuraev, L.N. Lipatov and V.S. Fadin, Zh. Eksp. Teor. Fiz. 71 (1976) 840 [Sov. Phys. JETP 44 (1976) 443]; 72 (1977) 377 [45 (1977) 199]; Ya.Ya. Balitskii and L.N. Lipatov, Sov. J. Nucl. Phys. 28 (1978) 822.

[5] J. Bartels, A. De Roeck, H. Lotter, Phys. Lett. B389 (1996) 742; S.J. Brodsky, F. Hautmann, D.E. Soper, Phys. Rev. D56 (1997) 6957; Phys. Rev. Lett. 78 (1997) 803; A. Bialas, W. Czyz, W. Florkowski, Eur. Phys. J. C2 (1998) 683; M. Boonekamp, A. De Roeck, C. Royon, S. Wallon, Nucl. Phys. B555 (1999) 540; J. Kwiecinski, L. Motyka, Acta Phys. Polon. B30 (1999) 1817; Eur. Phys. J. C18 (2000) 343.

[6] L.N. Lipatov, V.S. Fadin, Sov. J. Nucl. Phys. 50 (1989) 712.

[7] V.S. Fadin, R. Fiore, M.I. Kotsky, Phys. Lett. B359 (1995) 181.

[8] V.S. Fadin, R. Fiore, M.I. Kotsky, Phys. Lett. B387 (1996) 593.

[9] V.S. Fadin, L.N. Lipatov, Nucl. Phys. B406 (1993) 259.

[10] V.S. Fadin, R. Fiore, A. Quartarolo, Phys. Rev. D50 (1994) 5893; V.S. Fadin, R. Fiore, M.I. Kotsky, Phys. Lett. B389 (1996) 737; V.S. Fadin, L.N. Lipatov, Nucl. Phys. B477 (1996) 767; V.S. Fadin, M.I. Kotsky, L.N. Lipatov, Phys. Lett. B415 (1997) 97; V.S. Fadin, R. Fiore, A. Flachi, M.I. Kotsky, Phys. Lett. B422 (1998) 287.

[11] S. Catani, M. Ciafaloni, F. Hautman, Phys. Lett. B242 (1990) 97; Nucl. Phys. B366 (1991) 135; G. Camici and M. Ciafaloni, Phys. Lett. B386 (1996) 341; Nucl. Phys. B496 (1997) 305.

[12] V.S. Fadin, L.N. Lipatov, Phys. Lett. B429 (1998) 127.

[13] G. Camici and M. Ciafaloni, Phys. Lett. B430 (1998) 349. 
[14] V.T. Kim, L.N. Lipatov, G.B. Pivovariov, Proc. 29th Int. Symposium on Multiparticle Dynamics (ISMD99), Providence, Rhode Island, August 8-13, 1999, hep-ph/9911242; Proc. 8th Blois Workshop (EDS99), Protvino, Russia, June 27 - July 2, 1999, hep-ph/9911228; S.J. Brodsky, V.S. Fadin, V.T. Kim, L.N. Lipatov, G.B. Pivovariov, presented by V.T. Kim at PHOTONS-2001, Ascona, Switzerland, September 2-7, 2001, hep-ph/0111390.

[15] V.S. Fadin, Proceedings of the 6-th International Workshop on Deep Inelastic Scattering and QCD (DIS98), editors G.H. Coremans and R. Roosen, World Scientific, 1998, pp. 747-751, hep-ph/9807527.

[16] V.S. Fadin, R. Fiore, M.I. Kotsky and A. Papa, Phys. Rev. D61 (2000) 094006; Phys. Rev. D61 (2000) 094005.

[17] M. Ciafaloni, G. Rodrigo, JHEP 0005 (2000) 042, hep-ph/0004033.

[18] H. Cheng, T.T. Wu, Phys. Rev. D1 (1970) 2775; G.V. Frolov, V.N. Gribov, L.N. Lipatov, Phys. Lett. 31B (1970) 34; V.N. Gribov, L.N. Lipatov, G.V. Frolov, Yad. Fiz. 12 (1971) 994 [Sov. J. Nucl. Phys. 12 (1971) 543].

[19] V. Fadin, D. Ivanov, M. Kotsky, "New Trends in High-Energy Physics", ed. L.L. Jenkovszky, Kiev, 2000, pp. 190-194, hep-ph/0007119.

[20] J. Bartels, S. Gieseke, C.F. Qiao, Phys. Rev. D63 (2001) 056014, hep-ph/0009102.

[21] V. Fadin, D. Ivanov, M. Kotsky, Phys. Atom. Nucl. 65(8) (2002) 1513; hep-ph/0106099.

[22] J. Bartels, S. Gieseke, A. Kyrieleis, Phys. Rev. D65 (2002) 014006, hep-ph/0107152.

[23] J. Bartels, D. Colferai, S. Gieseke, A. Kyrieleis, hep-ph/0208130.

[24] V.S. Fadin, R. Fiore, Phys. Lett. B440 (1998) 359.

[25] V.S. Fadin, A.D. Martin, Phys. Rev. D60 (1999) 114008.

[26] V.S. Fadin, preprint BudkerINP 98-55, hep-ph/9807528, talk given at the International conference "LISHEP98", February 14-20, 1998, Rio de Janeiro, Brazil.

[27] V.S. Fadin, R. Fiore, Phys. Rev. D64 (2001) 114012, hep-ph/0107010.

[28] E. Kuraev, L. Lipatov, N. Merenkov, Phys. Lett. B47 (1973) 33. 